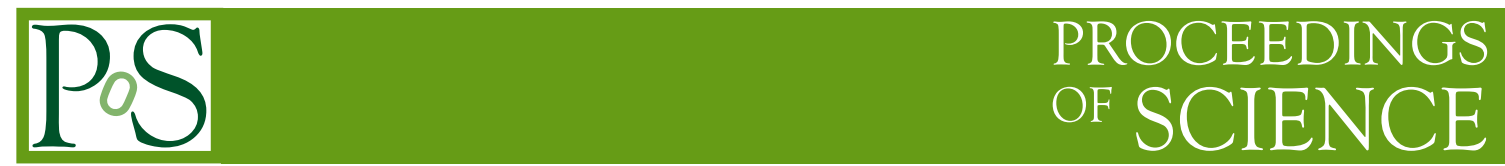

\title{
Aspects of Quantum Field Theory on Quantum Spacetime
}

\author{
Gherardo Piacitelli \\ SISSA - Via Bonomea 265, 34136 - Trieste (Italy) \\ E-mail: gherardo@piacitelli.org
}

We provide a minimal, self-contained introduction to the covariant DFR flat quantum spacetime, and to some partial results for the corresponding quantum field theory. Explicit equations are given in the Dirac notation.

Corfu Summer Institute on Elementary Particles and Physics - Workshop on Non Commutative Field Theory and Gravity,

September 8-12, 2010

Corfu Greece 


\section{Introduction}

At the time of the beautiful conference in Corfu, an extensive review on these and related topics had already been completed, which now is published as [1]. I will then take the opportunity of the present conference proceedings to complement that review with an explicit description of some applications to quantum field theory [2, 3, , 5] on DFR quantum spacetime [河]. The presentation is however self contained and addresses a possibly different audience. The second chapter partially overlaps with [1]], but contains more explicit equations. The third chapter contains a much more detailed description of results about quantum field theory than [1]. For the sake of dissemination, I will give explicit formulas in the Dirac notation, still favoured by many physicists. The reader interested in mathematical rigour is referred to the original papers.

In this first introductory chapter, we will very briefly recall some basics about local quantum field theory on the flat Minkowski spacetime in physical (i.e. 4) dimensions (section 1.1). Then we will discuss motivations for introducing non commutative coordinates, and their interpretation: in particular we will stress that they are NOT observables (section 1.2).

As for the comparison with other approaches to covariance, the interested reader may find a detailed discussion in [1]].

\subsection{A minimal account of local quantum field theory}

Relativistic quantum field theory results from merging the quantum theory of observables with the principle of locality: it is formulated in terms of operator fields $A(x)$, which can be regarded (up to idealisations) as sets of pointwise localised observables, labeled by the event $x$ at which they are localised; equivalently as more or less generalised "functions" of $\mathbb{R}^{4}$.

Einstein causality amounts to require the commutativity of any two observable fields $A(\cdot), B(\cdot)$ at spacelike distances, namely $[A(x+a), B(x)]=0$ for every $a$ spacelike.

There is a representation $U(\Lambda, a)$ of the Poincaré group. Then a (scalar) operator field $A(\cdot)$ is said covariant if $U(\Lambda, a) A(x) U(\Lambda, a)^{-1}=A(\Lambda x+a)$. Covariance is required so that every observable which is at rest in given frame can be described by any other equivalent observer (passive point of view); and also so that the measuring device can be displaced, rotated and boosted so to be brought at rest with respect to any other equivalent observer (active point of view). A covariant field, then, essentially describe a single device in all possible Lorentz frames.

For general reasons (spin-statistics theorem), possibly unobservable fields also must be allowed for, which either commute or anticommute at spacelike distances; for these and related reasons, covariance has to be generalised to multiplets of such fields. Here however we will confine ourselves with a theory generated by a single covariant Bosonic field.

The pairwise commuting generators $P^{\mu}$ of translations, defined by $U(I, a)=e^{i a P}$, fulfil the spectrum condition: $P^{0} \geqslant 0$ and $P^{0^{2}}-|\vec{P}|^{2} \geqslant 0$. In particular $H=P^{0}$ is the generator of time translations=time evolution, and is called the Hamiltonian (with respect to the given Lorentz frame).

The vacuum state $|\emptyset\rangle$ is defined as the unique translation invariant state (if symmetries are not spontaneously broken), thus fulfilling $P^{\mu}|\emptyset\rangle=0$. If the theory is defined by a single field $A(x)$, any state $|\Psi\rangle$ can be approximated by linear combinations of states of the form $A\left(x_{1}\right) \cdots A\left(x_{k}\right)|\emptyset\rangle$ (any $k$ ). 
By analogy with the Fock construction in the case of free fields, particles are usually told to be "carried" by fields: the statistics of particles (Bose/Fermi) is usually related with the kind of commutation rule at spacelike distance. Indeed a much deeper and gratifying understanding of statistics as a property of charged sectors (instead of particle-carrying fields) is available (see the review [6]); however we will not need such a theoretical deployment here, since we only will be concerned with toy models involving the simplest case (neutral Klein-Gordon free theory and perturbations).

Unfortunately, the free fields are the only known examples (in dimension 4) of theories fulfilling the above general requirements, and they only can describe a lifeless universe made of non interacting particles.

A perturbative approach has been developed, where the interacting dynamics is compared with the free dynamics, as an attempt to describe scattering experiments: incoming particles which are free in the far past and far away from the interaction region interact at finite times, and produce outgoing particles which are free and far away in the far future. The (formal) unitary operator $S$ which interpolates between the incoming and outgoing (asymptotic) free states is called the $S$ matrix. The perturbation series in powers of the coupling parameter is called the Dyson series.

This perturbative approach also is plagued by terrible problems; the formal equations defining the matrix elements of $S$ are affected by all kinds of divergences, and even conceptual obstructions to its existence ("Haag theorem") can be devised. Notwithstanding that, a clever strategy (driven by physical interpretation) for covariantly removing the most severe class of divergences (ultraviolet divergences) has been devised, called renormalisation. A theory is called renormalisable if the perturbation series for $S$ can be made to converge, at the cost of introducing infinite recursive subtractions depending from at most a small set of phenomenological parameters. Unfortunately the only known renormalisable model for which some indications about the limit $S$ can be obtained seems to give $S=I$, namely to converge to... a free theory.

Quite surprisingly, however, lowest order corrections in the perturbation theory of a physical theory (quantum electrodynamics) give experimentally verified predictions of incredible accuracy.

The interested reader will find more details on any standard textbook (e.g. [7]); the first two chapters of Haag's monograph [8] also provide a nice conceptual introduction, while the rest of the book is devoted to a mathematically advanced introduction to local quantum physics.

\subsection{Why should we quantise the coordinates?}

Notwithstanding the lack of non trivial well defined models, even perturbative, it would be way too much to dismiss the theory as a failure. The successful experimental predictions should instead be regarded as a strong indication that the illness of the theory is due to some conceptual ingredient which is still missing.

The ultraviolet divergences ultimately are a consequence of a too strict assumption about locality: the divergent expressions arise from interaction terms which are polynomials in the fields under the pointwise product. String theory arose as an attempt to replace points with less singular geometric objects. However this solution remains in the realm of classical geometry, and did not result as effective as it was hoped, so far.

The concept itself of space as a collection of infinitely small points dates back to Euclid and has never been challenged. Indeed, even Einstein observed that, as a logical consequence of accepting 
quantum physics, a quantum description of geometry would be conceptually necessary. Apart from this, if we take the Compton wavelength $\lambda_{C}(m)$ as the characteristic parameter driving the quantum behaviour of a point particle of mass $m$ and the Schwarzschild radius $\lambda_{C}(m)$ as characteristic of a pointwise source of gravitational field of mass $m$, the condition $\lambda_{S}(m) \sim \lambda_{C}(m)$ has solution $m \sim m_{P}$, the Planck mass, and $\lambda_{S}\left(m_{P}\right) \sim \lambda_{C}\left(m_{P}\right) \sim \lambda_{P}$, where $\lambda_{P} \sim 10^{-33} \mathrm{~cm}$ is the Planck length. This is a strong indication that quantum phenomena and gravitation come to interplay at the Planck scale, where the concept of point particle should be expected to break down.

As a first attempt in the direction of concrete physical models, it was proposed in [2] to provide a set of non commuting coordinates $q^{\mu}$ for the flat Minkowski spacetime, covariant under a unitary representation of the Poincaré group, in order to replace the usual localisation of quantum fields. The hope was to describe an intermediate model where the energy involved in single processes is sufficiently high to sense the quantum texture of spacetime; yet the density of processes is too small to produce curvature. Here we only will describe the solution, while we refer to the original paper or the less technical [9] for a derivation of the uncertainty and commutation relations. Earlier attempts are shortly discussed in [1].

Precisely as the components $x^{\mu}$ of the label $x$ of $A(x)$ are not observables, we are not going to interpret the selfadjoint operators $q^{\mu}$ as observables. To fix the interpretation, let us first describe a bit more precisely what happens in usual quantum field theory.

A localisation state on the classical spacetime may be described by a density $f(x) d x$ with $\int f(x) d x=1$, so that the average of the observable field $A(x)$ over the density gives the smeared field $A(f)=\int f(x) A(x) d x$. A sharp localisation ${ }^{1}$ is obtained by a delta: if $\delta_{a}(x) d x=\delta(x-$ a) $d x$, then $A\left(\delta_{a}\right)$ is the sharp localisation of the field $A(x)$ at $a$. We are led to think of $x$ as of a set of coordinate functions, of $f$ as a localisation state, and of the point $a$ as of a particular kind of localisation states.

In the case of quantum coordinates $q^{\mu}$, localisation states will be described by density matrices $\rho$, giving the expectation $\left\langle q^{\mu}\right\rangle_{\rho}=\operatorname{Tr}\left(\rho q^{\mu}\right)$. Vector states are a special cases where $\rho$ is of the form $|\xi\rangle\langle\xi|$. To avoid confusion, we anticipate that there will not be a direct correspondence between classical sharp localisation states and quantum vector states.

Let $A(x)$ be a quantum field on classical spacetime, taking values in the operators on some Hilbert space $\mathfrak{H}$; its DFR quantisation is the replacement of the classical coordinate functions $x^{\mu}$ with the quantum coordinate operators $q^{\mu}$ on the Hilbert space $\mathfrak{K}$, using a natural covariant generalisation of the Weyl prescription. If $A(x)=\int_{\mathbb{R}^{4}} d k \check{A}(k) e^{i k x}$, then

$$
\mathbf{A}(q)=\int_{\mathbb{R}^{4}} d k \check{A}(k) \otimes e^{i k q}
$$

as operators on $\mathfrak{H} \otimes \mathfrak{K}$, where $k x=k_{\mu} x^{\mu}$ and $k q=k_{\mu} q^{\mu}$. States on the second tensor factor of $\mathfrak{H} \otimes \mathfrak{K}$ describe a choice of the "localisation" of the field; then for any such choice we get an observable, whose physical states live in the first tensor factor. In other words, the expectation functional $\langle\cdot\rangle_{\rho}$, acting on the second tensor factor only, plays the same role as the density $f(x) d x$ :

\footnotetext{
${ }^{1}$ We will not concern ourselves here with the underlying technicalities (fields should be treated as generalised functions), since they are not relevant here.
} 
by analogy with the case of classical spacetime, we may introduce the notation $\mathbf{A}\left(\langle\cdot\rangle_{\rho}\right)$ for the partial expectation $\langle\mathbf{A}(q)\rangle_{\rho}$; then by linearity

$$
\mathbf{A}\left(\langle\cdot\rangle_{\rho}\right)=\int_{\mathbb{R}^{4}} d k \check{A}(k)\left\langle e^{i k q}\right\rangle_{\rho}=A\left(f_{\rho}\right),
$$

where

$$
f_{\rho}(x)=(2 \pi)^{-4} \int d k e^{-i k x}\left\langle e^{i k q}\right\rangle_{\rho} .
$$

It seems that the only effect of DFR quantisation is to select a certain subclass of test functions $f_{\rho}$ for the usual quantum fields. Indeed, the effect of quantisation manifests itself when products of fields are considered, as

$$
(\mathbf{A B})(q) \neq \mathbf{A}(q) \mathbf{B}(q) \text {. }
$$

Hence, interaction Lagrangians-which are usually defined in terms of local polynomials in the fields-have non trivial generalisations to DFR quantum spacetime.

It has to be stressed that, as far as the time component $x^{0}$ of classical localisation in quantum field theory has no interpretation as a time observable, so is for its quantum counterpart $q^{0}$.

\section{Covariant Quantum Geometry}

\subsection{The DFR quantum coordinates}

Consider the operators $P_{j}=-i \partial_{j}, Q_{j}=s_{j}$ on $L^{2}\left(\mathbb{R}^{4}\right), j=1,2,3,4$, which fulfil $P_{j} Q_{k}-$ $Q_{k} P_{j}=-i \delta_{j k}$. We then introduce the notations $X^{0}=P_{1}, X^{1}=P_{2}, X^{2}=Q_{1}, X^{3}=Q_{2}$. Finally, we set

$$
\mathfrak{K}=L^{2}(\mathscr{L}, d \Lambda) \otimes L^{2}\left(\mathbb{R}^{2}, d s_{1} d s_{2} d s_{3} d s_{4}\right),
$$

where $d \Lambda$ is the Haar measure of the Lorentz group $\mathscr{L}$. As usual, we associate to it a complete set of generalised kets

$$
|\Lambda\rangle|\underline{s}\rangle, \quad \Lambda \in \mathscr{L}, \underline{s}=\left(s_{1}, s_{2}, s_{3}, s_{4}\right) \in \mathbb{R}^{4},
$$

with bracket

$$
\left\{\left\langle\Lambda|\langle\underline{s}|\}\left\{\left|\Lambda^{\prime}\right\rangle\left|\underline{s}^{\prime}\right\rangle\right\}=\left\langle\Lambda \mid \Lambda^{\prime}\right\rangle\left\langle\underline{s} \mid \underline{s}^{\prime}\right\rangle=\delta\left(\Lambda^{-1} \Lambda^{\prime}\right) \delta^{(4)}\left(\underline{s}_{1}-\underline{s}_{1}^{\prime}\right),\right.\right.
$$

where integrals are taken with the measure $d \Lambda d \underline{s}$ and $\delta(\Lambda) d \Lambda$ is the purely atomic normalised measure on $\mathscr{L}$, concentrated on $I$.

We define the operators $q^{\mu}$ by their actions on the kets $|\Lambda\rangle|\xi\rangle$ :

$$
q^{\mu}|\Lambda\rangle|\xi\rangle=\lambda_{P}|\Lambda\rangle\left\{\Lambda^{\mu}{ }_{\nu} X^{\nu}|\xi\rangle\right\} .
$$

We may easily check that the operators $Q^{\mu \nu}$ defined by

$$
\left[q^{\mu}, q^{\nu}\right]=i \lambda_{P}^{2} Q^{\mu \nu}
$$

are simultaneously diagonalised by the kets $|\Lambda\rangle|\underline{s}\rangle$ :

$$
Q^{\mu \nu}|\Lambda\rangle|\underline{s}\rangle=\sigma(\Lambda)^{\mu \nu}|\Lambda\rangle|\underline{s}\rangle,
$$


where

$$
\sigma(I)=\left(\begin{array}{cccc}
0 & 0 & -1 & 0 \\
0 & 0 & 0 & -1 \\
1 & 0 & 0 & 0 \\
0 & 1 & 0 & 0
\end{array}\right)
$$

and

$$
\sigma(\Lambda)^{\mu \nu}=\Lambda_{\mu^{\prime}}^{\mu} \Lambda_{\nu^{\prime}}^{\nu} \sigma(I)^{\mu^{\prime} \nu^{\prime}}
$$

Moreover, by construction

$$
\left[q^{\mu}, Q^{\mu \nu}\right]=0 .
$$

We have a unitary representation $U(\cdot, 0)$ of the Lorentz group

$$
U(\Lambda, 0)|M\rangle|\underline{s}\rangle=|\Lambda M\rangle|\underline{s}\rangle
$$

it fulfils

$$
\begin{gathered}
U(\Lambda, 0)^{-1} q^{\mu} U(\Lambda, 0)=\Lambda^{\mu}{ }_{\nu} q^{\nu}, \\
U(\Lambda, 0)^{-1} Q^{\mu \nu} U(\Lambda, 0)=\Lambda_{\mu^{\prime}}^{\mu} \Lambda_{\mu^{\prime}} Q^{\mu^{\prime} \nu^{\prime}} .
\end{gathered}
$$

Note that, since $\sigma(\Lambda)_{\mu \nu} \sigma(\Lambda)^{\mu \nu}=\sigma(I)_{\mu \nu} \sigma(I)^{\mu \nu}=0$ is a Lorentz invariant, we have $Q^{\mu \nu} Q_{\mu \nu}|\Lambda\rangle|\xi\rangle=$ 0 identically and thus

$$
Q_{\mu \nu} Q^{\mu \nu}=0
$$

Analogously,

$$
Q_{\mu \nu}(* Q)^{\mu \nu}= \pm 4
$$

where $(* Q)^{\mu \nu}=(1 / 2) \epsilon^{\mu \nu \mu^{\prime} \nu^{\prime}} Q_{\mu^{\prime} \nu^{\prime}}$.

We finally make use of the remaining Schrödinger operators to construct the representation of translations. We first define

$$
\Pi^{0}=Q_{1}+Q_{3}, \quad \Pi^{1}=-Q_{2}-Q_{4}, \quad \Pi^{2}=P_{1}-P_{3}, \quad \Pi^{3}=P_{2}-P_{4},
$$

which fulfil $\left[\Pi^{\mu}, \Pi^{\nu}\right]=0$ and

$$
\left[\Pi^{\mu}, X^{\nu}\right]=i g^{\mu \nu}
$$

Then we define

$$
p^{\mu}\{|\Lambda\rangle|\xi\rangle\}=|\Lambda\rangle\left\{\Lambda^{\mu}{ }_{\nu} \Pi^{\nu}|\xi\rangle\right\}
$$

which fulfil

$$
\left[p^{\mu}, p^{\nu}\right]=0, \quad\left[p^{\mu}, q^{\nu}\right]=i g^{\mu \nu}
$$

It follows that

$$
U(\Lambda, a)=e^{i a p} U(\Lambda, 0)
$$

fulfils

$$
U(I, 0)=I, \quad U(\Lambda a) U(M, b)=U(\Lambda M, a+\Lambda b)
$$


and

$$
\begin{gathered}
U(\Lambda, a)^{-1} q^{\mu} U(\Lambda, a)=\Lambda^{\mu}{ }_{\nu} q^{\nu}+a^{\nu}, \\
U(\Lambda, a)^{-1} Q^{\mu \nu} U(\Lambda, a)=\Lambda_{\mu^{\prime}} \Lambda^{\mu}{ }_{\mu^{\prime}} Q^{\mu^{\prime} \nu^{\prime}} .
\end{gathered}
$$

Neither the coordinates $q^{\mu}$ nor the generators of translations $p^{\mu}$ have an interpretation as observables here. We are not aiming at a "more non commutative" quantum mechanics, but at a noncommutative localisation framework for quantum fields.

\subsection{States, uncertainties and optimal localisation}

The operators $q^{\mu}$ on $\mathfrak{K}$ generate all possible localisations through the choice of localisation states, namely expectation functionals of the form $\langle\cdot\rangle_{\rho}=\operatorname{Tr}(\rho \cdot)$ for some positive operator $\rho$ with $\operatorname{Tr}(\rho)=1$ (a density matrix). Such a state describes fuzzy localisation around the point $a \in \mathbb{R}^{4}$ given by $a^{\mu}=\left\langle q^{\mu}\right\rangle_{\rho}$, with variance $\Delta_{\rho}\left(q^{\mu}\right)^{2}=\left\langle\left(q^{\mu}-\left\langle q^{\mu}\right\rangle_{\rho}\right)^{2}\right\rangle_{\rho}$.

By Heisenberg-like arguments, it can be proved that the uncertainties $\Delta_{\rho}\left(q^{\mu}\right)$ fulfil the bounds ${ }^{2}$

$$
\begin{gathered}
\Delta_{\rho}\left(q^{0}\right)\left(\Delta_{\rho}\left(q^{1}\right)+\Delta_{\rho}\left(q^{2}\right)+\Delta_{\rho}\left(q^{3}\right)\right) \geqslant \lambda_{P}^{2}, \\
\Delta_{\rho}\left(q^{1}\right) \Delta_{\rho}\left(q^{2}\right)+\Delta_{\rho}\left(q^{2}\right) \Delta_{\rho}\left(q^{3}\right)+\Delta_{\rho}\left(q^{3}\right) \Delta_{\rho}\left(q^{1}\right) \geqslant \lambda_{P}^{2} .
\end{gathered}
$$

The proof can be found in [2].

In the classical case, localisation states arise as probability densities $f(x) d x$ on $\mathbb{R}^{4}$. Points correspond to sharp densities $\delta_{a}(x)=\delta(x-a) d x$. Since $f$ 's may be rewritten as $f(\cdot)=\int d a f(a) \delta_{a}(\cdot)$, they can be thought of as convex linear combinations of $\delta_{a}$ 's (up to taking limits of integral sums). Hence probability densities are the classical analogues of statistical mixtures and sharp densities are the analogues of pure states.

Since however the set of operators $q^{\mu}$ is not irreducible (by Schur's lemma: the commutators $\left[q^{\mu}, q^{\nu}\right]$ are not multiples of the identity), the usual identification of statistical mixtures with non trivial density matrices, and of pure states with vector states, breaks down: there are indeed vector states which are not pure! The pure states are those described by those particular vector states, which are of the form $|\Lambda\rangle|\xi\rangle$, namely they must in particular be eigenkets of the commutators $\left[q^{\mu}, q^{\nu}\right]$.

In view of the large scale limit, one would like to have a notion of states with the best possible localisation properties; then these states could be used to define interactions with the smallest possible deviations from usual locality. Indeed, an important condition is that the usual local quantum theory should appear as a limiting case of the new theory (large scale limit).

A moment's though, however, shows that this is impossible, at least in such naive terms. Because of the uncertainty relations, states are extended objects in some sense, and as such they always can be delocalised at wish by suitable Lorentz boosts. No such notion as a covariant class of states with optimal localisation can be devised. This is the fundamental reason why all more or less trivial generalisations of local interactions have led to break Lorentz covariance so far. It can be regarded as an indication that the noncommutative notion which should give locality in the large scale limit is non trivial and still missing.

\footnotetext{
${ }^{2}$ There is no reason to expect such bounds to be form-covariant: indeed the uncertainty $\Delta_{\rho}$ is not linear in its argument, so that $\left.\Delta_{\rho}\left(\Lambda_{\nu}^{\mu} q^{\nu}\right) \neq \Lambda_{\nu}^{\mu} \Delta_{\rho}\left(q^{\nu}\right)\right)$ in general.
} 
The best one can do is to define well localised states with respect to some choice of a class of reference frames which are pairwise related by a Galilei transformation (no Lorentz boosts). A suitable criterion, then, is to select those states $\rho$ which minimise $\sum_{\mu} \Delta_{\rho}\left(q^{\mu}\right)^{2}$. It is clear that these states are essentially given in terms of the coherent states of the Schrödinger operators $X^{\mu}$ used in the construction of the coordinate operators.

Consider first a state of the form $|I\rangle\left|\eta_{0}\right\rangle$ where $\left|\eta_{0}\right\rangle$ is the normalised ground state of the harmonic oscillator for the Schrödinger operators ${ }^{3}$; then the sum of the squares of the corresponding uncertainties attains the minimum

$$
\sum_{\mu} \Delta\left(q^{\mu}\right)^{2}=\lambda_{P}^{2} \sum_{\mu} \Delta\left(X^{\mu}\right)^{2}=\lambda_{P}^{2}\left(\Delta\left(Q_{1}\right)^{2}+\Delta\left(Q_{2}\right)^{2}+\Delta\left(P_{1}\right)^{2}+\Delta\left(P^{2}\right)^{2}\right)=\lambda_{P}^{2} ;
$$

a state with these properties is said an optimal localisation state. Note that in the states described here above, the coordinates have expectation 0 , so that $\Delta\left(q^{\mu}\right)$ equals the expectation of $q^{\mu 2}$ in such states.

Next, we consider the state $|R\rangle\left|\eta_{0}\right\rangle$ where $R \in O(3) \subset \mathscr{L}$. For such a state, the coordinates still have expectation in the origin, and

$$
\frac{\left\{\left\langleR\left|\left\langle\eta_{0}\right|\right\} \sum_{\mu}\left(q^{\mu}\right)^{2}\left\{|R\rangle\left|\eta_{0}\right\rangle\right\}\right.\right.}{\left\{\left\langleR\left|\left\langle\eta_{0}\right|\right\}\left\{|R\rangle\left|\eta_{0}\right\rangle\right\}\right.\right.}=\lambda_{P}^{2}\left\langle\eta_{0}\left|\sum_{\mu} R^{\mu}{ }_{\nu} X^{\nu 2}\right| \eta_{0}\right\rangle=\lambda_{P}^{2}\left\langle\eta_{0}\left|\sum_{\mu} X^{\mu 2}\right| \eta_{0}\right\rangle=\lambda_{P}^{2} ;
$$

we used that $R$ is orthogonal and that $\sum_{\mu} X^{\mu 2}=2 H_{0}$ where $H_{0}=\frac{1}{2}\left(P_{1}^{2}+P_{2}^{2}+Q_{1}^{2}+Q_{2}^{2}\right)$. So we still find the ground state of the harmonic oscillator. It follows that $|R\rangle\left|\eta_{a}\right\rangle$ also is an optimal localisation state, with expectation in the origin.

Finally for every $a \in \mathbb{R}^{4}$ and any $R \in O(3) \subset \mathscr{L}$ we define $\left|\eta_{a}\right\rangle$ by setting $|R\rangle\left|\eta_{a}\right\rangle=$ $U(I, a)|R\rangle\left|\eta_{0}\right\rangle$; by unitarity, $|R\rangle\left|\eta_{a}\right\rangle$ is an optimal localisation state, but now $q$ is expected at $a$.

Indeed, it can be shown (see [2]) that the states described above and their superpositions (with same $a$ ) are precisely all the possible optimal localisation states. We have

$$
\left\{\left\langleR\left|\left\langle\eta_{a}\right|\right\} e^{i k q}\left\{|R\rangle\left|\eta_{a}\right\rangle\right\}=e^{i k a} e^{-\frac{1}{2} \lambda_{P}^{2} \sum_{\mu}\left(k^{\mu}\right)^{2}} .\right.\right.
$$

\subsection{Independent localisation events}

The standard way of constructing the coordinates $q_{j}^{\mu}$ of independent events is via tensor products, taking

$$
q_{j}^{\mu}=I \otimes \cdots \otimes I \otimes q^{\mu} \otimes I \otimes \cdots \otimes I \quad\left(q^{\mu} \text { in the } j^{\text {th }} \text { position }\right),
$$

so that the commutation relations are of the form

$$
\left[q_{j}^{\mu}, q_{k}^{\nu}\right]=i \delta_{j k} \lambda_{P}^{2} Q_{j}^{\mu \nu}
$$

If we take the usual definition of tensor product, we get

$$
Q_{j}^{\mu \nu}=I \otimes \cdots \otimes Q^{\mu \nu} \otimes \cdots \otimes I \quad\left(Q^{\mu \nu} \text { in the } j^{\text {th }} \text { position }\right) .
$$

\footnotetext{
${ }^{3}$ Actually, we should add a degeneration label $\kappa$ and write $\left|\eta_{0}, \kappa\right\rangle$; since however this degeneracy is only an artefact of the amplification which is used to implement translations, we shall omit the label $\kappa$.
} 
However, again due to the reducibility of the set $\left\{q^{\mu}\right\}$ of operators, a different construction which also deserves the name of tensor product is possible, for which the commutators fulfil

$$
Q_{1}^{\mu \nu}=Q_{2}^{\mu \nu}=\cdots
$$

and the $j$ dependence of $Q^{\mu \nu}$ in (2.10) can be dropped [3]. The idea is to define the direct product of kets "pointwise in $\Lambda^{\text {": }}$

$$
\left\{|\Lambda\rangle\left|\xi_{1}\right\rangle\right\}\left\{|\Lambda\rangle\left|\xi_{2}\right\rangle\right\} \cdots\left\{|\Lambda\rangle\left|\xi_{n}\right\rangle\right\}=|\Lambda\rangle\left|\xi_{1}\right\rangle\left|\xi_{2}\right\rangle \cdots\left|\xi_{n}\right\rangle
$$

These kets span the Hilbert space $L^{2}(\mathscr{L}) \otimes L^{2}\left(\mathbb{R}^{4}\right) \otimes \cdots \otimes L^{2}\left(\mathbb{R}^{4}\right)$ (usual direct product). On such kets, we define the operator

$$
F_{j}|\Lambda\rangle\left|\xi_{1}\right\rangle\left|\xi_{2}\right\rangle \cdots\left|\xi_{n}\right\rangle=|\Lambda\rangle\left|\xi_{j}\right\rangle\left|\xi_{1}\right\rangle\left|\xi_{2}\right\rangle \cdots\left|\xi_{j-1}\right\rangle\left|\xi_{j+1}\right\rangle \cdots\left|\xi_{n}\right\rangle,
$$

which exchange $\left|\xi_{1}\right\rangle$ with $\left|\xi_{j}\right\rangle$. Finally, accordingly with the new definition of direct product,

$$
\begin{gathered}
\left.q_{1}^{\mu}|\Lambda\rangle\left|\xi_{1}\right\rangle\left|\xi_{2}\right\rangle \cdots\left|\xi_{n}\right\rangle=\left\{q^{\mu}|\Lambda\rangle\left|\xi_{1}\right\rangle\right\}\right\rangle\left|\xi_{2}\right\rangle \cdots\left|\xi_{n}\right\rangle, \\
q_{j}^{\mu}=F_{j} q^{\mu} F_{j},
\end{gathered}
$$

which are easily checked to have the desired properties.

Both choices of $\otimes$ give covariant coordinates. In particular for the construction described above-which we will adopt from now on - the representation of the Poincare group is

$$
U(\Lambda, 0)|M\rangle\left|\xi_{1}\right\rangle\left|\xi_{2}\right\rangle \cdots\left|\xi_{n}\right\rangle=|\Lambda M\rangle\left|\xi_{1}\right\rangle\left|\xi_{2}\right\rangle \cdots\left|\xi_{n}\right\rangle,
$$

and

$$
U(\Lambda, a)^{-1} q_{j}^{\mu} U(\Lambda, a)=\Lambda_{\nu}^{\mu} q_{j}^{\nu}+a^{\mu} .
$$

\subsection{How close can independent events come to?}

One reason why the direct product "taken pointwise over $\Lambda$ " is preferable when constructing the coordinates of many independent events is that it leads to a natural generalisation of the classical concept of localising independent events at the same point [3]. This can be used for example when a function $f\left(x_{1}, x_{2}, \ldots, x_{n}\right)$ of $n$ events is evaluated on the diagonal set, giving a function $g(x)=f(x, x, \ldots, x)$ of one event only.

Define the operator

$$
\bar{q}^{\mu}=\frac{1}{\sqrt{n}}\left(q_{1}^{\mu}+\cdots+q_{n}^{\mu}\right) .
$$

As a consequence of (2.11),

$$
\left[\bar{q}^{\mu}, q_{j}^{\nu}-q_{k}^{\nu}\right]=0,
$$

namely $\bar{q}^{\mu}$ is statistically independent from the differences of any two events (this would not be the case if the ordinary construction of the direct product were taken instead).

Note then that every $q_{j}^{\mu}$ can be written as a linear combination of $\bar{q}^{\mu}$ and the differences $q_{j}^{\mu}-q_{k}^{\mu}$ :

$$
q_{j}^{\mu}=\frac{1}{\sqrt{n}} \bar{q}^{\mu}+\frac{1}{n} \sum_{k}\left(q_{j}^{\mu}-q_{k}^{\mu}\right) .
$$


The above remarks suggest to consider a different realisation of the same commutation relations, using one more tensor factor.

We define

$$
\tilde{q}_{j}^{\mu}=\frac{1}{\sqrt{n}} q^{\mu} \otimes \underbrace{I \otimes \cdots \otimes I}_{n \text { factors }}+\frac{1}{n} \sum_{k} I \otimes\left(q_{j}^{\mu}-q_{k}^{\mu}\right) .
$$

as operators on $\underbrace{\mathfrak{R} \otimes \cdots \otimes \mathfrak{K}}_{n+1 \text { factors }}$ (direct products taken "pointwise in $\Lambda$ ”). It is clear by construction that

$$
\left[\tilde{q}_{j}^{\mu}, \tilde{q}_{k}^{\nu}\right]=i \lambda_{P}^{2} \delta_{j k} Q^{\mu \nu},
$$

where $I \otimes Q^{\mu \nu}$ is identified with $Q^{\mu \nu}$ according to (2.11). Moreover the average coordinate is

$$
\frac{1}{n} \sum_{j} \tilde{q}_{j}=\frac{1}{\sqrt{n}} q^{\mu} \otimes \underbrace{I \otimes \cdots \otimes I}_{n \text { factors }},
$$

which commutes with the $\tilde{q}_{j}^{\mu}$,s.

The unitary representation

$$
\tilde{U}(\Lambda, a)=\underbrace{U(\Lambda, a) \otimes \cdots \otimes U(\Lambda, a)}_{n+1 \text { factors }}
$$

fulfils

$$
\tilde{U}(\Lambda, a)^{-1} \tilde{q}_{j}^{\mu} \tilde{U}(\Lambda, a)=\Lambda_{\nu}^{\mu} \tilde{q}_{j}^{\nu}+a^{\mu} .
$$

We now want to set all difference $\tilde{q}_{j}-\tilde{q}_{k}$ to thei minimum value at once, compatibly with the uncertainty relations.

Before giving the general construction, we first discuss an easier, less general construction, which allows to highlight the main point.

We choose a state $|R\rangle\left|\eta_{a}\right\rangle$ with optimal localisation, as discussed in section 2.3; here $R$ is an element of $O(3) \subset \mathscr{L}$ and $\eta_{a}$ is a coherent state.

Observe now that the direct product $|\Psi\rangle$ of $n$ copies of this state gives, with our particular definition of direct product,

$$
|\Psi\rangle=\underbrace{\left\{|R\rangle\left|\eta_{a}\right\rangle\right\} \cdots\left\{|R\rangle\left|\eta_{a}\right\rangle\right\}}_{n \text { factors }}=|R\rangle \underbrace{\left|\eta_{a}\right\rangle\left|\eta_{a}\right\rangle \cdots\left|\eta_{a}\right\rangle}_{n \text { factors }} .
$$

We can use it to define a partial expectation on the last $n$ tensor factor.

The components of the separation

$$
\delta_{j k} \tilde{q}^{\mu}=\frac{\tilde{q}_{j}^{\mu}-\tilde{q}_{k}^{\mu}}{\sqrt{2}}
$$

between two independent events still fulfil the same relations as the coordinates themselves:

$$
\left[\delta_{j k} \tilde{q}^{\mu}, \delta_{j k} \tilde{q}^{\nu}\right]=i \lambda_{P}^{2} Q^{\mu \nu}
$$


Moreover, the partial expectation over the state $|\Psi\rangle$ (acting on the last $n$ tensor factors) gives

$$
\begin{gathered}
\left\langle\Psi\left|\delta_{j k} \tilde{q}^{\mu}\right| \Psi\right\rangle=0, \\
\sum_{\mu} \Delta\left(\delta_{j k} \tilde{q}^{\mu}\right)^{2}=\sum_{\mu}\left\langle\Psi\left|\left(\delta_{j k} \tilde{q}^{\mu}\right)^{2}\right| \Psi\right\rangle=\lambda_{P}^{2},
\end{gathered}
$$

and the latter is precisely the property selecting the states with optimal localisation; note that, as expected, the choice of $a$ is irrelevant. Hence we may say that this partial expectation has the effect of setting the differences $\delta \tilde{q}_{j k}$ as close to zero as possible, compatibly with the uncertainty relations. We regard this as a quantum generalisation of the classical operation of setting $x_{1}=x_{2}=\ldots$.

This is almost what we want; the only problem is that there is no need to restrict to a particular joint eigenspace of the $Q^{\mu \nu}$ 's, namely the one corresponding to the projection on $|R\rangle$. It is sufficient to restrict to the sum of all joint eigenspaces of the $Q^{\mu \nu}$ 's which correspond to orthogonal transformations.

To do this, we split the above operation in two steps. We first define the orthogonal projection $E$ which sends $|\Lambda\rangle\left|\xi_{1}\right\rangle\left|\xi_{2}\right\rangle \cdots\left|\xi_{n}\right\rangle$ to 0 if $\Lambda$ contains a Lorentz boost, and leaves it unchanged otherwise. We have

$$
\begin{gathered}
{\left[\tilde{q}_{j}^{\mu}, E\right],} \\
{[\tilde{U}(R, a), E]=0, \quad R \in O(3), a \in \mathbb{R}^{4} .}
\end{gathered}
$$

In other words, $E$ is the biggest possible projection which commutes with all $q_{j}^{\mu}$,s and is stable under orthogonal transformations. We then restrict our coordinates $\tilde{q}_{j}^{\mu}$ to operators acting on the range $L^{2}(O(3)) \otimes L^{2}\left(\mathbb{R}^{4(n+1)}\right)$ of $E$, and afterwards we take the partial expectation on the state $\left|\eta_{a}\right\rangle \cdots\left|\eta_{a}\right\rangle$ ( $n$ factors) acting on the last $n$ direct factors. The resulting map $\mathbb{E}$ has its range in the operators on $L^{2}(O(3)) \otimes L^{2}\left(\mathbb{R}^{4}\right)$, and has the following properties:

$$
\begin{gathered}
\mathbb{E}\left[\delta_{j k} \tilde{q}^{\mu}\right]=0, \\
\mathbb{E}\left[e^{i k_{\mu} \delta_{j k} \tilde{q}^{\mu}}\right]=e^{-\lambda_{P}^{2} \frac{1}{2} \sum_{\mu}\left(k^{\mu}\right)^{2}}
\end{gathered}
$$

as multiples of the identity operator.

Moreover

$$
\mathbb{E}\left[\frac{\tilde{q}_{1}^{\mu}+\cdots+\tilde{q}_{n}^{\mu}}{n}\right]|R\rangle|\xi\rangle=\frac{\lambda_{P}}{\sqrt{n}}|R\rangle\left\{R^{\mu}{ }_{\nu} X^{\nu}|\xi\rangle\right\},
$$

which defines new operators

$$
\dot{q}^{\mu}=\mathbb{E}\left[\frac{q_{1}+\cdots+q_{n}}{n}\right]
$$

on $L^{2}(O(3)) \otimes L^{2}\left(\mathbb{R}^{4}\right)$ with the nice property that the corresponding commutators

$$
\left[\dot{q}^{\mu}, \dot{q}^{\nu}\right]=i\left(\frac{\lambda_{P}}{\sqrt{n}}\right)^{2} \dot{Q}^{\mu \nu}
$$

induce the same uncertainty relations of the initial coordinates, but with the Planck length scaled by $\sqrt{n}$. This is precisely what one would expect of the statistical behaviour of a mean of independent stochastic variables. In the large $n$ limit, the average coordinate of many events becomes deterministic. 
Note that the coordinates $\dot{q}^{\mu}$ are covariant under orthogonal transformations and translations:

$$
\dot{U}(R, a)^{-1} \dot{q}^{\mu} \dot{U}(R, a)=R^{\mu}{ }_{\nu} \dot{q}^{\nu}+a^{\mu}, \quad R \in O(3) \subset \mathscr{L}, a \in \mathbb{R}^{4},
$$

where the representation $\dot{U}(R, a)$ is obtained by restricting each unitary operator $U(R, a)$ to $L^{2}(O(3)) \otimes$ $L^{2}\left(\mathbb{R}^{4}\right)$.

Finally, the map $\mathbb{E}$ is covariant in the sense that

$$
\dot{U}(R, a)^{-1} \mathbb{E}[\cdot] \dot{U}(R, a)=\mathbb{E}\left[\tilde{U}(R, a)^{-1} \cdot \tilde{U}(R, a)\right], \quad R \in O(3) \subset \mathscr{L}, a \in \mathbb{R}^{4} .
$$

We will use this map to define a quantum generalisation of the Wick product in section 3.5, where we will need the explicit form of $\mathbb{E}\left[e^{i \sum_{j} k_{j} q_{j}}\right]$ which we will now compute.

We first map $e^{i \sum_{j} k_{j} q_{j}}$ (which acts on kets of the form $|\Lambda\rangle\left|\xi_{1}\right\rangle \cdots\left|\xi_{n}\right\rangle$ ), into $e^{i \sum_{j} k_{j} \tilde{q}_{j}}$ (which acts on kets of the form $\left.|\Lambda\rangle|\xi\rangle\left|\xi_{1}\right\rangle \cdots\left|\xi_{n}\right\rangle\right)$.

We observe that

$$
\sum_{j} k_{j} \tilde{q}_{j}=\left(\frac{1}{\sqrt{n}} \sum_{j} k_{j}\right) q \otimes \underbrace{I \otimes \cdots \otimes I}_{n \text { factors }}+I \otimes \sum_{j}\left(k_{j}-\frac{1}{n} \sum_{l} k_{l}\right) q_{l}) .
$$

acting on $|R\rangle|\xi\rangle\left|\xi_{1}\right\rangle \cdots\left|\xi_{n}\right\rangle$. It follows that

$$
e^{i \sum_{j} k_{j} \tilde{q}_{j}}=\left(e^{\frac{i}{\sqrt{n}}\left(\sum_{j} k_{j}\right) q}\right) \otimes\left(e^{i \sum_{j}\left(k_{j}-\frac{1}{n} \sum_{l} k_{l}\right) q_{l}}\right) .
$$

where the direct product is taken "pontwise in $\Lambda$ ".

Now we restrict to the range of the projection $E$. We do that simply by restricting ourselves from now on to kets $|R\rangle$ with $R \in O(3) \subset \mathscr{L}$.

We take the partial expectation of $e^{i \sum_{j} k_{j} \tilde{q}_{j}}$ (restricted to the range of $E$ ) over the last $n$ factors, using a state $\left|\eta_{0}\right\rangle \cdots\left|\eta_{0}\right\rangle$ where $\eta_{0}$ is the ground state of the harmonic oscillator: this gives

$$
\begin{aligned}
\mathbb{E}\left[e^{i \sum_{j} k_{j} q_{j}}\right] & =e^{i \sum_{j} k_{j} \dot{q}}\{\underbrace{\left\langle\eta_{0}\right| \cdots\left\langle\eta_{0}\right|}_{n \text { factors }}\} \sum_{j}\left(k_{j}-\frac{1}{n} \sum_{l} k_{l}\right) q_{l}\{\underbrace{\left|\eta_{0}\right\rangle \cdots\left|\eta_{0}\right\rangle}_{n \text { factors }}\}= \\
& =e^{-\lambda_{P}^{2} \frac{1}{2}\left(\sum_{j}\left|k_{j}\right|^{2}-\sum_{j l} k_{j} \cdot k_{l}\right)} e^{i \sum_{j} k_{j} \dot{q}}
\end{aligned}
$$

where $h \cdot k=\sum_{\mu=0}^{3} k^{\mu} h^{\mu},|k|^{2}=\sqrt{k \cdot k}$, and we recall that $\dot{q}^{\mu}$ is the restriction of $q^{\mu} / \sqrt{n}$ to $L^{2}(O(3)) \otimes L^{2}\left(\mathbb{R}^{4}\right)$.

\subsection{Distance, area and volume operators}

In the framework of the universal differential calculus of [10], we may define the differential of coordinates as

$$
d q^{\mu}=q^{\mu} \otimes I-I \otimes q^{\mu} .
$$

This provides another reason why the construction of $\otimes$ which we used in section 2.3 is preferable: if it is used in the definition of $d$, then $d Q^{\mu \nu}=0$, which is compatible with the interpretation of $Q^{\mu \nu}$ as an independent background. 
If the universal calculus is used alone, the realisation of the commutation relations by operators on the Hilbert space plays little role. An interesting way of making the differential calculus to interplay with operator products is to use the operator product instead of the tensor product when multiplying differentials with each other [11], e.g.

$$
\begin{aligned}
d q^{\mu} \cdot d q^{\nu} & =\left(q^{\mu} \otimes I-I \otimes q^{\mu}\right) \cdot\left(q^{\nu} \otimes I-I \otimes q^{\nu}\right)= \\
& =q^{\mu} \otimes q^{\nu} \otimes I-q^{\mu} \otimes I \otimes q^{\nu}-I \otimes q^{\mu} q^{\nu} \otimes I+I \otimes q^{\mu} \otimes q^{\nu}
\end{aligned}
$$

So the product of two differentials is a combination of products of operator living on the 3 -fold tensor product of the one-event state space; which is consistent with the interpretation of $d q$ as a "segment" with two extreme events, the product of two differentials describing the "join" of two such "segments" at the same event.

In particular, a very simple generalisation of the usual definitions of area and 3- and 4-volume operators can be given [11]. For example, the 4-volume operator is defined as

$$
V=\epsilon_{\mu \nu \rho \sigma} d q^{\mu} \cdot d q^{\nu} \cdot d q^{\rho} \cdot d q^{\sigma}
$$

which lives in the 5-fold tensor product, and indeed one needs five events to give a hypercube in four dimensions.

The resulting operator is not selfadjoint, as a consequence of the commutators which show up when exchanging the order of the "vertexes". Quite unexpectedly, $V$ is normal, namely $V V^{*}=$ $V^{*} V$. The phase operator appearing in the polar decomposition of the 4-volume operator can be regarded as a quantum generalisation of the sign describing the orientation.

The 4-volume operator is very complicated; yet its spectrum can be computed and is found to be $\left\{(n \sqrt{5} \pm 2+i a) \lambda_{P}^{4}: n \in \mathbb{Z}, a \in \mathbb{R}\right\}$. In particular the absolute value of the 4-volume operator is bounded below by $(\sqrt{5}-2) \lambda_{P}^{4} \approx .23 \lambda_{P}^{4}$. We refer to the original paper [11] for the details of the computation.

\subsection{The $\star$-product}

Let

$$
\Sigma=\{\sigma(\Lambda): \Lambda \in \mathscr{L}\}
$$

according to the notation (2.2).

Provided that the integrals exist, we may associate to each complex function $f(\sigma ; x)$ of $\Sigma \times \mathbb{R}^{4}$ the operator

$$
\begin{aligned}
f(Q ; q)\{|\Lambda\rangle|\xi\rangle\} & =|\Lambda\rangle\left\{\int d k \check{f}(\sigma(\Lambda) ; k) e^{i \lambda_{P}(\Lambda X) k}|\xi\rangle\right\}= \\
& =|\Lambda\rangle\left\{\int d k \check{f}(\sigma(\Lambda) ; k) e^{i \lambda_{P} X\left(\Lambda^{-1} k\right)}|\xi\rangle\right\},
\end{aligned}
$$

where the Fourier transformation acts on $f(\sigma ; \cdot)$ for every $\sigma$ fixed. Note that, if $f$ does not depend explicitly on $\sigma$, the above is simply $\int d k \check{f}(k) e^{i k q}$; if, otherwise, $f$ does not depend explicitly on $x$, the above is the usual function $f(Q)$ of the sixteen pairwise commuting operators $Q^{\mu \nu}$. 
Now we consider the product of two such operators

$$
\begin{aligned}
& f(Q ; q) g(Q ; q)\{|\Lambda\rangle|\xi\rangle\}= \\
& \quad=|\Lambda\rangle\left\{\int d h d k \check{f}(\sigma(\Lambda) ; h) \check{g}(\sigma(\Lambda) ; k) e^{i \lambda_{P} X\left(\Lambda^{-1} h\right)} e^{i \lambda_{P} X\left(\Lambda^{-1} k\right)}|\xi\rangle\right\}
\end{aligned}
$$

By definition the operators $X^{\mu}$ (defined at the beginning of section 2.1) fulfil the commutation relations $\left[X^{\mu}, X^{\nu}\right]=\sigma(I)^{\mu \nu}$, where $\sigma(I)$ is given by (2.2a). The BCH formula implies

$$
e^{i \lambda_{P} h X} e^{i \lambda_{P} k X}=e^{-\frac{i}{2} \lambda_{P}^{2} \sigma(I)^{\mu \nu} h_{\nu} k_{\nu}} e^{i(h+k) X}
$$

substituting this in (2.25), we get

$$
f(Q ; q) g(Q ; q)\{|\Lambda\rangle|\xi\rangle\}=|\Lambda\rangle\left\{\int d h d k(\check{f} \tilde{\times} \check{g})(\sigma(\Lambda) ; k) e^{\left.i \lambda_{P} k X\right)}|\xi\rangle\right\},
$$

where

$$
(\check{f} \tilde{\times} \check{g})(\sigma ; k)=\int d h \check{f}\left(\lambda_{P}^{2} \sigma ; h\right) \check{g}\left(\lambda_{P}^{2} \sigma ; k-h\right) e^{-\frac{i}{2} \lambda_{P}^{2} \sigma^{\mu \nu} h_{\nu} k_{\nu}},
$$

and antisymmetry of $\sigma$ has been used.

Defining now

$$
(f \star g)(\sigma ; x)=\int d k(\check{f} \tilde{\times} \check{g})(\sigma ; k) e^{i k x},
$$

we get

$$
f(Q ; q) g(Q ; q)=(f \star g)(Q ; q) .
$$

Note that, even in the case when $f$ and $g$ do not explicitly depend on $\sigma$, their $\star$-product does.

For the explicit expression of $\star$ and its (complicate) relationship with the Moyal expansion, see [1]. The $\star$-product here only plays an ancillary role. Its only use in this paper is the following. To every $f(Q ; q)$ and $t \in \mathbb{R}$ we can associate the operator

$$
\int_{x^{0}=t} d^{3} x f(Q ; x)
$$

on $L^{2}(\mathscr{L})$ whose action is

$$
\int_{x^{0}=t} d^{3} x f(Q ; x)|\Lambda\rangle=\int_{x^{0}=t} d^{3} x f(\sigma(\Lambda) ; x)|\Lambda\rangle .
$$

This map is positive, in the sense that it maps $f(Q ; q)^{*} f(Q ; q)$ (which is a positive operator) to another positive operator (see [四, Sec. 5] for the proof). We only will need the $\star$-product in the case of operators of the form $f_{1}(Q ; q) \cdots f_{n}(Q ; q)=\left(f_{1} \star \cdots \star f_{n}\right)(Q ; q)$, which the above map sends into $\int_{x^{0}=t}\left(f_{1} \star \cdots \star f_{n}\right)(Q ; x)$. 


\section{Quantum Field Theory on Quantum Spacetime}

\subsection{The Klein-Gordon field on classical spacetime}

To fix the notations, we briefly recall the standard definition of the massive free scalar spin 0 field (see any standard textbook, e.g. [7]).

We establish a Dirac bracket notation which defines the (Fock) Hilbert space $\mathfrak{H}$. The complete system of kets

$$
\begin{array}{lr}
|\emptyset\rangle, & n=0, \\
\left|\vec{k}_{1}, \ldots, \vec{k}_{n}\right\rangle, & n=1,2, \ldots, \quad \vec{k}_{j} \in \mathbb{R}^{3}
\end{array}
$$

fulfils the normalisation condition

$$
\begin{aligned}
& \left\langle\vec{h}_{1}, \vec{h}_{2} \ldots, \vec{h}_{m} \mid \vec{k}_{1}, \vec{k}_{2}, \ldots, \vec{k}_{n}\right\rangle= \\
& =\frac{\delta_{n m}}{n !} \sum_{\pi} \delta^{(3)}\left(\vec{h}_{1}-\vec{k}_{\pi_{1}}\right) \delta^{(3)}\left(\vec{h}_{2}-\vec{k}_{\pi_{2}}\right) \cdots \delta^{(3)}\left(\vec{h}_{n}-\vec{k}_{\pi_{n}}\right),
\end{aligned}
$$

where the sum runs over all permutations $\pi$ of $(1, \ldots, n)$, and the $\delta^{(3)}$ 's are defined with respect to the usual translation invariant measure $d^{3} k$ on $\mathbb{R}^{3}$. In particular for any such $\pi$ we have $\left|\vec{k}_{1}, \ldots, \vec{k}_{n}\right\rangle=\left|\vec{k}_{\pi_{1}}, \ldots, \vec{k}_{\pi_{n}}\right\rangle$. The ket $|\emptyset\rangle$ is called the vacuum state.

The creation and annihilation operators

$$
\begin{aligned}
a^{\dagger}(\vec{k})\left|\vec{k}_{1}, \ldots, \vec{k}_{n}\right\rangle & =\sqrt{n+1}\left|\vec{k}, \vec{k}_{1}, \ldots, \vec{k}_{n}\right\rangle \\
a(\vec{k})\left|\vec{k}_{1}, \ldots, \vec{k}_{n}\right\rangle & =\sqrt{\frac{1}{n}} \sum_{j} \delta^{(3)}\left(k-k_{j}\right)\left|\vec{k}_{1}, \ldots, \vec{k}_{j-1}, \vec{k}_{j+1}, \ldots, \vec{k}_{n}\right\rangle
\end{aligned}
$$

fulfil

$$
\begin{gathered}
a^{\dagger}(k)=a(k)^{*}, \\
{\left[a(\vec{h}), a^{\dagger}(\vec{k})\right]=\delta^{(3)}(\vec{h}-\vec{k}),} \\
{[a(\vec{h}), a(\vec{k})]=\left[a^{\dagger}(\vec{h}), a^{\dagger}(\vec{k})\right]=0,} \\
a(\vec{k})|\emptyset\rangle=0 .
\end{gathered}
$$

The scalar Klein-Gordon field is defined as

$$
\varphi(x)=\int_{\mathbb{R}^{4}} d^{4} k \check{\varphi}(k) e^{i k x}
$$

where

$$
\check{\varphi}\left(k^{0}, \vec{k}\right)=\sqrt{\frac{2\left|k^{0}\right|}{(2 \pi)^{3}}} \delta\left(k^{2}-m^{2}\right)\left\{\theta\left(-k^{0}\right) a(-\vec{k})+\theta\left(k^{0}\right) a^{\dagger}(\vec{k})\right\} .
$$

Note that to each 3 -vector $\vec{k}$ there is a unique 4 -vector $\tilde{k}$ belonging to the upper mass shell (namely fulfilling $k^{0}>0$ and $\left.k^{2}=k^{0^{2}}-|\vec{k}|^{2}=m^{2}\right)$. Explicitly, $\tilde{k}=\left(\omega_{m}(\vec{k}), \vec{k}\right)$, where $\omega_{m}(\vec{k})=$ $\sqrt{m^{2}+|\vec{k}|^{2}}$. 
Since $\square e^{i k x}=-k^{2} e^{i k x}$ (where $\square=\partial_{\mu} \partial^{\mu}$ ), we have

$$
\left(\square+m^{2}\right) \varphi=0
$$

There is a representation $\mathscr{U}(\Lambda, a)$ of the restricted Poincaré group $\mathscr{P}_{+}^{\uparrow}$ (where only Lorentz transformations preserving the arrow of time and the orientation of space are allowed) by unitary operators on $\mathfrak{H}$ : its action is

$$
\begin{gathered}
\mathscr{U}(\Lambda, a)|\emptyset\rangle=|\emptyset\rangle, \\
\mathscr{U}(\Lambda, a)\left|\vec{k}_{1}, \ldots, \vec{k}_{n}\right\rangle=e^{i\left(\sum_{j} \Lambda \tilde{k}_{j}\right) a}\left|\overrightarrow{\Lambda \tilde{k}_{1}}, \overrightarrow{\Lambda \tilde{k}_{2}}, \ldots, \overrightarrow{\Lambda \tilde{k}_{n}}\right\rangle
\end{gathered}
$$

The notation is a bit involved, let us describe what happens: we start from a 3 -vector $\vec{k}$, we construct the corresponding on-shell 4-vector $\tilde{k}$, we apply to it the Lorentz transformation $\Lambda$ giving the 4vector $\Lambda \tilde{k}$, whose space part is $\overrightarrow{\Lambda \tilde{k}}$. Note that, since $\Lambda$ preserves the time arrow, $\Lambda \tilde{k}$ is in the upper mass shell, too.

By construction,

$$
\mathscr{U}(\Lambda, a) a^{\dagger}(\vec{k}) \mathscr{U}(\Lambda, a)^{-1}=e^{i(\Lambda \tilde{k}) a} a^{\dagger}(\overrightarrow{\Lambda \tilde{k}})
$$

It follows that ${ }^{4}$

$$
\begin{aligned}
& \mathscr{U}(\Lambda, a) \check{\varphi}(k) \mathscr{U}(\Lambda, a)^{-1}=e^{i(\Lambda k) a} \check{\varphi}(\Lambda k), \\
& \left.\mathscr{U}(\Lambda, a) \varphi(x) \mathscr{U}(\Lambda, a)^{-1}=\varphi(\Lambda x+a)\right)
\end{aligned}
$$

where $(\Lambda, a) \in \mathscr{P}_{+}^{\uparrow}$.

The spectrum condition holds, namely the generators $P^{\mu}$ of translations defined by $\mathscr{U}(I, a)=$ $e^{i a P}$ fulfil $P_{\mu} P^{\mu} \geqslant 0$ and $P^{0} \geqslant 0$. In particular we have $P^{\mu}|\emptyset\rangle=0$ and

$$
P^{\mu}\left|\vec{k}_{1}, \ldots, \vec{k}_{n}\right\rangle=\sum_{j} \tilde{k}_{j}^{\mu}\left|\vec{k}_{1}, \ldots, \vec{k}_{n}\right\rangle ;
$$

note that any finite sum $\sum_{j} \tilde{k}_{j}^{\mu}$ is contained in the convex hull of the upper mass shell of mass $m$.

It is noteworthy for our purposes that the Hamiltonian $H_{0}=P^{0}$ of the free field takes the form

$$
H_{0}=\int_{x^{0}=t} d^{3} x \mathscr{H}_{0}(x)
$$

where

$$
\mathscr{H}_{0}(x)=\frac{1}{2} \int_{x^{0}=0} d^{3} x:\left(\partial^{0} \varphi\right)(x)^{2}-\left(\partial^{0} \partial^{0} \varphi\right)(x) \varphi(x):
$$

and the double dots indicate normal (Wick) ordering of annihilations and creations.

\footnotetext{
${ }^{4}$ To check these computations the matrix notation for Lorentz matrices can be useful: the Lorentzian product $k a=$ $k_{\mu} a^{\mu}$ may be written as $K^{t} G A$, where $K, A$ are column vectors, $t$ is matrix transposition, and $G=\operatorname{diag}(1,-1,-1,-1)$ is the metric. Then conservation of the metric is $\Lambda G \Lambda^{t}=G$. From this and $G^{2}=I$ follows $\Lambda^{-1}=G \Lambda^{t} G$, which in turn gives (back to usual notations) $k(\Lambda a)=\left(\Lambda^{-1} k\right) a$.
} 


\subsection{DFR quantisation of the Klein-Gordon field}

According to the discussion of the introduction,

$$
\varphi(q)=\int d k \check{\varphi}(k) \otimes e^{i k q}
$$

as an operator on $\mathfrak{H} \otimes \mathfrak{K}$, where $\mathfrak{H}$ is the Fock space and the coordinates $q^{\mu}$ are operators on $\mathfrak{K}$. In particular, for $\left|k_{1}, \ldots, k_{n}\right\rangle \in \mathfrak{H}$ and $|\Lambda\rangle|\xi\rangle \in \mathfrak{K}$,

$$
\varphi(q)\left\{\left|k_{1}, \ldots, k_{n}\right\rangle|\Lambda\rangle|\xi\rangle\right\}=\int d k\left\{\check{\varphi}(k)\left|k_{1}, \ldots, k_{n}\right\rangle\right\}\left\{e^{i k q}|\Lambda\rangle|\xi\rangle\right\} .
$$

By abuse of notations, we still denote by $\mathscr{U}, U$ the representations of Poincaré transformations acting non trivially on the first and second tensor factor of $\mathfrak{H} \otimes \mathfrak{K}$, respectively.

We find

$$
\begin{aligned}
\mathscr{U}(\Lambda, a) \varphi(q) \mathscr{U}(\Lambda, a)^{-1} & =\int d k\left(\mathscr{U}(\Lambda, a) \check{\varphi}(k) \mathscr{U}(\Lambda, a)^{-1}\right) \otimes e^{i k q}= \\
& =\int d k e^{i(\Lambda k) a} \check{\varphi}(\Lambda k) \otimes e^{i k q}= \\
& =\int d h \check{\varphi}(h) \otimes e^{i h(\Lambda q+a)}= \\
& =\int d h \check{\varphi}(h) \otimes\left(U(\Lambda, a)^{-1} e^{i h q} U(\Lambda, a)\right)= \\
& =U(\Lambda, a)^{-1} \varphi(q) U(\Lambda, a) .
\end{aligned}
$$

Hence the DFR quantisation of a free quantum field is covariant. Note that the above result can be given the simpler form

$$
\mathscr{U}(\Lambda, a) \varphi(q) \mathscr{U}(\Lambda, a)^{-1}=\varphi(\Lambda q+a) .
$$

Since translations are unitarily implemented, we also have derivatives; we find that the DFR quantisation commutes with taking derivatives:

$$
\lim _{\lambda \rightarrow 0} \frac{1}{\lambda}\left(\varphi\left(q+\lambda e^{\mu}\right)=\left(\partial_{\mu} \varphi\right)(q),\right.
$$

where $e^{\mu}$ is the $\mu^{\text {th }}$ canonical basis vector of $\mathbb{R}^{4}$.

Hence, the DFR quantised free field fulfils the Klein-Gordon equation:

$$
\left(\square+m^{2}\right) \varphi(q)=0 .
$$

The partial expectation of $\varphi(q)$ over a state with optimal localisation around $a \in \mathbb{R}^{4}$ gives, according to 1.1 , the free field operator on the Fock space $\mathfrak{H}$, smeared with a Gaussian:

$$
\begin{aligned}
F_{a}=\left\{\left\langleR\left|\left\langle\eta_{a}\right|\right\} \varphi(q)\left\{|R\rangle\left|\eta_{a}\right\rangle\right\}\right.\right. & =\int d k \check{\varphi}(k)\left\{\left\langleR\left|\left\langle\eta_{a}\right|\right\} e^{i k q}\left\{|R\rangle\left|\eta_{a}\right\rangle\right\}=\right.\right. \\
& =\frac{1}{(2 \pi)^{2}} \int d x \varphi(x) e^{-\frac{|x-a|^{2}}{2 \lambda_{P}^{2}}}
\end{aligned}
$$


where $|x-a|^{2}=\sum_{\mu}\left(x^{\mu}-a^{\mu}\right)^{2}$. It follows that

$$
\left[F_{a}, F_{a+x}\right] \propto i \frac{\lambda_{P}}{|\vec{x}|}\left(e^{-\frac{1}{8 \lambda_{P}^{2}}\left(|\vec{x}|-x^{0}\right)^{2}}-e^{-\frac{1}{8 \lambda_{P}^{2}}\left(|\vec{x}|+x^{0}\right)^{2}}\right)
$$

which falls off as a Gaussian in spacelike dimensions (as a function of $x$ ), and converges to the usual commutator function in the large scale limit.

\subsection{The DFR perturbative setup}

The basic idea of the DFR perturbative setup is to construct an effective non local quantum field theory on the classical spacetime: the underlying idea is the following: there are incoming and outgoing free fields on the classical spacetime, describing free particles: since they do not interact, they "do not know" that the spacetime is quantum. When interaction takes place, the quantum texture of spacetime enters in the game, and this is taken into account by a nonlocal deformation of the interaction Lagrangian.

A key remark is that

$$
\boldsymbol{H}_{0}(Q)=\int_{x^{0}=t} d^{3} x \mathscr{H}_{0}(q)=H_{0} \otimes I,
$$

namely the free Hamiltonian is left essentially unchanged by the Weyl quantisation, apart from the $\otimes I$ which reminds that, while $H_{0}$ is an operator on the Fock space $\mathfrak{H}$, its quantisation is an operator on $\mathfrak{H} \otimes \mathfrak{L}^{2}(\mathscr{L})$.

Since $Q^{\mu \nu}$ is unaffected by time translations, we have

$$
e^{i t \boldsymbol{H}_{0}(Q)} \boldsymbol{\varphi}\left(q^{0}, q^{1}, q^{2}, q^{3}\right) e^{-i t \boldsymbol{H}_{0}(Q)}=\boldsymbol{\varphi}\left(q^{0}+t, q^{1}, q^{2}, q^{3}\right) .
$$

Free fields are essentially unaffected by the DFR quantisation.

This remark led the authors of [2] to continue this analogy, and define a nonlocal generalisation of the interaction Lagrangian $: \varphi(x)^{n}:$ as $: \varphi(q)^{n}$ :, which can be written equivalently as : $(\varphi \star \cdots \star \varphi$ : $(Q ; q)$. Then, by the remarks at the end of section 2.6, it makes sense to consider

$$
\boldsymbol{H}_{I, \star}(Q ; t)=\int_{x^{0}=t} d^{3} x:(\varphi \star \cdots \star \varphi):(Q, x),
$$

again as a (formal) operator on $\mathfrak{H} \otimes \mathfrak{L}^{2}(\Lambda)$, and we add the subscript $\star$ to keep track of the choice of the noncommutative Wick product.

At a certain point, we will have to take a partial expectation so to obtain an effective interaction term $H_{I, \star}^{\text {eff }}(t)$ as an operator on the Fock space $\mathfrak{H}$; in other words, we have to integrate out the $Q$ dependence. This is necessary to obtain a scattering matrix which interpolates the incoming and outgoing free fields, which live on the Fock space $\mathfrak{H}$ alone; indeed, physical intuition suggests that the noncommutativity gets averaged out over large distances without interactions. However, let us leave this apart for a while. 
Mimicking usual QFT, we formally define the limit $\boldsymbol{S}_{\star}(Q)=\boldsymbol{U}_{\star}(Q ; \infty,-\infty)$ of the unitary evolution semigroup $\boldsymbol{U}_{\star}(Q ; t, s)$, which fulfils

$$
\begin{gathered}
\boldsymbol{U}_{\star}(Q ; t, t)=\boldsymbol{I}, \\
\boldsymbol{U}_{\star}(Q ; s, t) \boldsymbol{U}_{\star}(Q ; t, u)=\boldsymbol{U}_{\star}(Q ; s, u), \\
\boldsymbol{U}_{\star}(Q ; s, t)^{-1}=\boldsymbol{U}_{\star}(Q ; t, s),
\end{gathered}
$$

and solves the evolution equation

$$
\frac{\partial \boldsymbol{U}_{\star}}{\partial t}(Q ; t, s)=i \boldsymbol{H}_{I, \star}(Q ; t) \boldsymbol{U}_{\star}(Q ; t, s)
$$

Its formal solution is given by the Dyson series

$$
\boldsymbol{S}_{\star}(Q)=I+\sum_{N=1}^{\infty} \frac{i^{N}}{N !} \int d t_{1} \cdots d t_{N} T\left[\boldsymbol{H}_{I, \star}\left(t_{1}\right), \ldots, \boldsymbol{H}_{I, \star}\left(t_{N}\right)\right],
$$

where $T$ means that the product of the $\boldsymbol{H}_{I, \star}\left(t_{j}\right)$ 's is taken in the order of decreasing times, namely

$$
\begin{aligned}
& T\left[\boldsymbol{H}_{I, \star}\left(t_{1}\right), \ldots, \boldsymbol{H}_{I, \star}\left(t_{N}\right)\right]= \\
& =\sum_{\pi} \theta\left(t_{\pi_{1}}-t_{\pi_{2}}\right) \theta\left(t_{\pi_{2}}-t_{\pi_{3}}\right) \cdots \theta\left(t_{\pi_{n-1}}-t_{\pi_{n}}\right) \boldsymbol{H}_{I, \star}\left(t_{\pi_{1}}\right) \boldsymbol{H}_{I, \star}\left(t_{\pi_{2}}\right) \cdots \boldsymbol{H}_{I, \star}\left(t_{\pi_{n}}\right)
\end{aligned}
$$

where $\theta$ is Heaviside's step function and the sum runs over the permutations of $(1, \ldots, n)$.

Note that the time ordered product is taken with respect to the labels $t_{j}$ at which the space integral is taken.

The matrix elements of $S_{\star}$ (as an operator on the Fock space, times $L^{2}(\mathscr{L})$ ) are of the form

$$
\left\{\left\langleh_{1}, \ldots, h_{m}|\langle\Lambda|\} \boldsymbol{S}_{\star}\left\{\left|k_{1}, \ldots, k_{n}\right\rangle|M\rangle\right\}=\delta\left(\Lambda^{-1} M\right)\left\langle h_{1}, \ldots, h_{m}\left|S_{\star}(\sigma(\Lambda))\right| k_{1}, \ldots, k_{n}\right\rangle\right.\right.
$$

where $S_{\star}(\sigma)$ is a non local, non causal scattering matrix on the usual Fock space. Note that the dependence on $\lambda_{P}$ is hidden in the product $\star$. Indeed, for every $\sigma \in \Sigma$ we have the formal limit

$$
S_{\star}(\sigma) \underset{\lambda_{P} \rightarrow 0}{\longrightarrow} S_{\mathrm{loc}},
$$

where $S_{\text {loc }}$, is the local, causal scattering matrix of the (non renormalised) $\varphi_{4}^{n}$ theory on the classical Minkowski spacetime.

The situation is much alike that of a bundle of non local theories over $\Sigma$. To integrate out this dependence, one would like to take a partial expectation on the second tensor factor with some Lorentz invariant state in $L^{2}(\mathscr{L})$. Unfortunately, no such state exists, essentially because the Lorentz group is not amenable ${ }^{5}$. The most symmetric choice is to take the rotation invariant state described by the characteristic function of the set $O(3) \subset \mathscr{L}$, which is square summable since $O(3)$ is compact. The partial expectation of $\boldsymbol{S}_{\star}$ on such state defines an effective scattering matrix

$$
S_{\star}^{\mathrm{eff}}=\int_{\Sigma^{(1)}} d \sigma S_{\star}(\sigma)
$$

\footnotetext{
${ }^{5}$ This means that there is no left-invariant mean of the functions of $\mathscr{L}$. Of course there is the Haar measure $d \Lambda$, but a mean should send, by definition, the constant function 1 to 1 , while $\int d \Lambda 1=\infty$.
} 
on the Fock space $\mathfrak{H}$, where $\Sigma^{(1)}=\{\sigma(R): R \in O(3) \subset \mathscr{L}\}$ and $d \sigma$ is the invariant measure on $\Sigma$ induced by the Haar measure of $\mathscr{L}$, normalised so that $\Sigma^{(1)}$ has measure 1 . This gives a theory covariant under rotations, but not under Lorentz boosts ${ }^{6}$.

If we perform a shameless exchange $\int d \sigma \sum_{N}=\sum_{N} \int d \sigma$ in the Dyson series, the above would be the same as starting from the beginning with a non local effective interaction term

$$
H_{I, \star}^{\mathrm{eff}}(t)=\int_{\Sigma^{(1)}} d \sigma \int_{x_{0}=0} d^{3} x: \varphi \star \cdots \star \varphi:(\sigma ; x)
$$

on the Fock space $\mathfrak{H}$.

Since all these developments are formal, there is room for experimentation about the order of summation and integrations. Choosing to integrate out the $\sigma$ dependence separately at each vertex after having performed Wick reduction, the effective scattering matrix was found ultraviolet finite in the $\varphi_{4}^{3}$ theory [12].

It is clear that, even if the effective theory is regular, its large scale limit will reproduce the non renormalised theory. Hence finite renormalisation would anyway be necessary, where the finite subtractions should diverge in the large scale limit so to reproduce the infinite subtractions of usual renormalisation.

\subsection{Unitarity and Feynman diagrams}

Since the Hamiltonian is formally selfadjoint, the scattering matrix is formally unitary, and no violations of unitarity should be expected. The violations discussed in the literature may be regarded as a consequence of an inconsistent prescription for the time ordering (as pointed out in [13]).

Indeed, for a local theory, the second order contribution to the Dyson series is

$$
S_{\mathrm{loc}, 2}=\frac{1}{2} \iint d s d t T\left[H_{I}^{\mathrm{loc}}(s), H_{I}^{\mathrm{loc}}(t)\right] .
$$

Since the time ordering does nothing to a pointwise product of $n$ fields, namely $T[\varphi(x), \ldots, \varphi(x)]=$ $\varphi(x)^{n}$, the above can be written as

$$
\begin{aligned}
S_{\mathrm{loc}, 2} & =\frac{1}{2} \iint d s d t \int_{x^{0}=s} d^{3} x \int_{y^{0}=t} d^{3} y T\left[: \varphi(x)^{n}:,: \varphi(y)^{n}:\right]= \\
& =\frac{1}{2} \int d^{4} x \int d^{4} y T\left[: \varphi(x)^{n}:,: \varphi(y)^{n}:\right],
\end{aligned}
$$

\footnotetext{
${ }^{6}$ Some authors claim to obtain a fully covariant interacting theory by means of a Lorentz invariant measure $W(\theta) d \theta$, where $d \theta$ is the invariant measure on the space $\mathcal{T}$ of real second rank antisymmetric tensors. Unfortunately, such a Lorentz invariant measure must be of the form $W(\theta) d \theta=w\left(\theta^{\mu \nu} \theta_{\mu \nu},\left(\theta^{\mu \nu}(* \theta)_{\mu \nu}\right)^{2}\right) d \theta$ for some $w(a, b)$. Hence

$$
\int_{\mathcal{T}} W(\theta) d \theta f(\theta)=\int_{-\infty}^{\infty} d a \int_{0}^{\infty} d b w(a, b) \int_{\mathcal{T}_{a, b}} d \theta_{a, b} f(\theta),
$$

where $\left.\mathcal{T}_{a, b}=\left\{\theta: \theta^{\mu \nu} \theta_{\mu \nu}=a,\left(\theta^{\mu \nu}(* \theta)_{\mu \nu}\right)^{2}=b\right)\right\}$ and $d \theta_{a, b}$ is the measure induced by $d \theta$ on $\mathcal{T}_{a, b}$. For every $a, b$, $\int_{\mathcal{T}_{a, b}} d \theta_{a, b}=\infty$, hence this measure, though Lorentz invariant, does not define a mean. The situation is similar to the absence of a translation invariant normalised measure on the line: there's no such thing as the expected position of a particle equally distributed on the line.
} 
where the time ordering is with respect to the times $x^{0}, y^{0}$. In other words, the time ordering can be brought inside integrals.

For the non local theory described by the interaction term $H_{I, \star}^{\text {eff }}(t)$, this cannot be done, since the latter is of the form

$$
H_{I, \star}^{\mathrm{eff}}=\int d a_{1} \cdots d a_{n} w\left(a_{1}, \ldots, a_{n} ; t\right): \varphi\left(a_{1}\right) \cdots \varphi\left(a_{n}\right):
$$

for some totally symmetric kernel $w$, depending on the time parameter $t$ (and implicitly on $\lambda_{P}$ ). The time ordering is taken with respect to such time parameters, not to the time components $a_{j}^{0}$ of the integration variables.

Indeed, using the proper integral form of $\star$, there's no such object as a " $T\left[\varphi\left(x_{1}\right) \star \cdots \star \varphi\left(x_{n}\right)\right]$ ". But if instead one illegally uses the Moyal expansion of $\star$, then he/her is misled to think that the noncommutative product is defined pointwise, since the twist only contains derivatives; in which case one would find " $T[\varphi(x) \star \cdots \star \varphi(x)]=\varphi(x) \star \cdots \star \varphi(x)$ " (false) and again could safely bring the time ordering inside the integral (false). This is one possible mechanism to obtain the violations of unitarity. For a general discussion of the drawbacks of the Moyal expansion, see [1].

Upon inserting the Dyson series in the Gell-Mann\&Low formula, the usual diagrammatic expansion may be used, with minor modifications to the rules. To every vertex, associate a factor $w\left(a_{1}, \ldots, a_{n} ; t\right) d a_{1} \cdots d a_{n}$; to every line originating from that vertex and labeled by $x$ pick a factor $\frac{1}{i}\left(\Delta_{+}\left(x-a_{j}\right) \theta\left(x^{0}-t\right)+\Delta_{+}\left(a_{j}-x\right) \theta\left(t-x^{0}\right)\right.$; between any line connecting the vertex with another vertex $w\left(b_{1}, \ldots, b_{n} ; s\right) d b_{1} \cdots d b_{n}$, pick a factor $\frac{1}{i}\left(\Delta_{+}\left(b_{k}-a_{l}\right) \theta(s-t)+\Delta_{+}\left(a_{l}-n_{k}\right) \theta(t-s)\right.$; and for any two external lines labeled by $x, y$, pick a usual Stueckelberg-Feynman propagator $\Delta_{S F}\left(a_{j}-b_{k}\right)$. See [5] for a detailed discussion.

\subsection{Quantum Wick Products and ultraviolet regularity}

It is peculiar of the process of generalisation that equivalent procedures may have inequivalent generalisations. Consider $n$ functions $f_{j}\left(x_{j}\right)$ of independent variables, and define $F\left(x_{1}, \ldots, x_{n}\right)=$ $f_{1}\left(x_{1}\right) \cdots f_{n}\left(x_{n}\right)$. The evaluation of $F$ at coincident points may be described either as 1) setting $x_{1}=x_{2}=\ldots=x_{n}=x$, or 2 ) evaluate the pointwise product of functions $f_{1} \cdots f_{n}$ at $x$. The equivalence of these two procedures is summarised by

$$
f_{1}(x) \cdots f_{n}(x)=\left(f_{1} \cdots f_{n}\right)(x),
$$

which is so natural that on first sight we do not even notice the point.

The non commutative generalisation of the "product strategy" is to replace the pointwise product with the product of quantised functions, or equivalently with the star product, so that one obtains

$$
\left(f_{1} \star \cdots \star f_{n}\right)(Q ; q) \text {. }
$$

This was used in the definition of non local Wick product $: \varphi \star \cdots \star \varphi$ : discussed in the preceding section.

A non commutative generalisation of the "bring independent events to the same place" strategy instead may be given in terms of the map $\mathbb{E}$ discussed in section 2.4, and gives a different result. It was used in [14, 3] to obtain a different generalisation of Wick product. 
The idea is to define the quantum wick product as

$$
: \varphi(\dot{q})^{n}:_{Q}=\mathbb{E}\left[: \varphi\left(q_{1}\right) \cdots \varphi\left(q_{n}\right):\right],
$$

where it is understood that $\mathbb{E}$ acts on the localisation part.

Since

$$
\begin{aligned}
: \varphi\left(q_{1}\right) \cdots \varphi\left(q_{n}\right): & =\int d^{4} k_{1} \cdots d^{4} k_{n}: \check{\varphi}\left(k_{1}\right) \cdots \check{\varphi}\left(k_{n}\right): \otimes e^{i k_{1} q_{1}} \cdots e^{i k_{n} q_{n}}= \\
& =\int d^{4} k_{1} \cdots d^{4} k_{n}: \check{\varphi}\left(k_{1}\right) \cdots \check{\varphi}\left(k_{n}\right): \otimes e^{i \sum_{j} k_{j} q_{j}},
\end{aligned}
$$

we get by linearity and (2.20)

$$
\begin{aligned}
\mathbb{E}\left[: \varphi\left(q_{1}\right) \cdots \varphi\left(q_{n}\right):\right] & =\int d^{4} k_{1} \cdots d^{4} k_{n}: \check{\varphi}\left(k_{1}\right) \cdots \check{\varphi}\left(k_{n}\right): \otimes \mathbb{E}\left[e^{i \sum_{j} k_{j} q_{j}}\right]= \\
& =\int d^{4} k_{1} \cdots d^{4} k_{n} e^{-\lambda_{P}^{2} \frac{1}{2}\left(\sum_{j}\left|k_{j}\right|^{2}-\sum_{j l} k_{j} \cdot k_{l}\right)}: \check{\varphi}\left(k_{1}\right) \cdots \check{\varphi}\left(k_{n}\right): \otimes e^{i\left(\sum_{j} k_{j}\right) \dot{q}} .
\end{aligned}
$$

Standard Fourier theory now gives

$$
\mathbb{E}\left[: \varphi\left(q_{1}\right) \cdots \varphi\left(q_{n}\right):\right]=: \underbrace{\varphi \star \cdots \star \varphi}_{n \text { factors }}:_{Q}(\dot{q})
$$

where

$$
\begin{aligned}
& : \underbrace{\varphi \star \cdots \star \varphi}_{n \text { factors }}::_{Q}(x)= \\
& =\frac{n^{2}}{(2 \pi)^{8(n-1)}} \int d a_{1} \cdots d a_{n}: \varphi\left(x+a_{1}\right) \cdots \varphi\left(x+a_{n}\right): e^{-\frac{1}{2 \lambda_{P}^{2}} \sum_{j}\left|a_{j}\right|^{2}} \delta^{(4)}\left(\frac{1}{n \lambda_{P}} \sum_{j} a_{j}\right)
\end{aligned}
$$

With this definition, we obtain an interaction term of the form

$$
\boldsymbol{H}_{I, \mathscr{G}}(t)=H_{I, \mathscr{G}}^{\mathrm{eff}}(t) \otimes \dot{I}
$$

as an operator on $\mathfrak{H} \otimes L^{2}(O(3))$, where

$$
\begin{aligned}
& H_{I, \mathscr{G}}^{\mathrm{eff}}(t)=\int_{x^{0}=0} d^{3} x: \varphi \star \cdots \star \varphi:_{Q}(x)= \\
& =\frac{n^{2}}{(2 \pi)^{8(n-1)}} \int_{x^{0}=t} d^{3} x \int d a_{1} \cdots d a_{n}: \varphi\left(x+a_{1}\right) \cdots \varphi\left(x+a_{n}\right): \\
& \quad e^{-\frac{1}{2 \lambda_{P}^{2}} \sum_{j}\left|a_{j}\right|^{2}} \delta^{(4)}\left(\frac{1}{n \lambda_{P}} \sum_{j} a_{j}\right) .
\end{aligned}
$$

Here covariance under Lorentz boosts is broken by the map $\mathbb{E}$. The resulting effective interaction term $\boldsymbol{H}_{I, \mathscr{G}}^{\text {eff }}(t)$ does not depend explicitly on $\sigma \in \Sigma^{(1)}$. Hence the average over the invariant 
measure gives precisely $H_{I, \mathscr{G}}^{\text {eff }}(t)$ which defines a non local, non causal scattering matrix on the Fock space $\mathfrak{H}$.

Note that the only effect of noncommutativity here is to naturally reproduce a particular recipe for the so called "point-split regularisation" in terms of a $\mathscr{G}$ aussian kernel. The resulting $H_{I, \mathscr{G}}^{\text {eff }}(t)$ is completely free of ultraviolet divergences. However, the same remarks apply here about the need of finite renormalisation, as in the end of section 3.3 .

\section{References}

[1] G. Piacitelli, Quantum Spacetime: a Disambiguation, SIGMA 6 (2010) 073, [1 004 .5261].

[2] S. Doplicher, K. Fredenhagen, and J. E. Roberts, The Quantum structure of space-time at the Planck scale and quantum fields, Commun. Math. Phys. 172 (1995) 187-220, hep-th/0303037.

[3] D. Bahns, S. Doplicher, K. Fredenhagen, and G. Piacitelli, Ultraviolet Finite Quantum Field Theory on Quantum Spacetime, Commun. Math. Phys. 237 (2003) 221-241, hep-th/0301100].

[4] D. Bahns, S. Doplicher, K. Fredenhagen, and G. Piacitelli, Field Theory on Noncommutative Spacetimes: Quasiplanar Wick Products, Phys. Rev. D71 (2005) 025022, hep-th/0408204.

[5] G. Piacitelli, Non local theories: New rules for old diagrams, JHEP 08 (2004) 031, [hep-th/0403055].

[6] S. Doplicher, The Principle of Locality. Effectiveness, fate and challenges, J. Math. Phys. 51 (2010) 015218, [0911.5136].

[7] J. D. Bjorken and S. D. Drell, Relativistic quantum fields. McGraw-Hill, New York-St. Louis-S. Francisco-Toronto-London-Sydney, 1965.

[8] R. Haag, Local quantum physics: Fields, particles, algebras. Springer, Berlin, 1992.

[9] S. Doplicher, Spacetime and fields, a quantum texture, in New Developments in Fundamental Interaction Theories (J. Lukierski and J. Rembielinski, eds.), vol. 589, AIP, 2001. hep-th/0105251.

[10] M. Dubois-Violette, Dérivations et calcul differentiel non commutatif, C. R. Acad. Sci. Paris 307 (1988) 403-408.

[11] D. Bahns, S. Doplicher, K. Fredenhagen, and G. Piacitelli, Quantum Geometry on Quantum Spacetime: Distance, Area and Volume Operators, 1005.2130.

[12] D. Bahns, Ultraviolet finiteness of the averaged Hamiltonian on the noncommutative Minkowski space, hep-th/0405224.

[13] D. Bahns, S. Doplicher, K. Fredenhagen, and G. Piacitelli, On the unitarity problem in space/time noncommutative theories, Phys. Lett. B533 (2002) 178-181, hep-th/0201222.

[14] G. Piacitelli, Normal Ordering of Operator Products on Quantum Spacetime and Quantum Field Theory. PhD thesis, Università di Padova, 2002. 